\title{
Imagerie à l'échelle atomique par holographie de fluorescence
}

\author{
S. Marchesini, G. Faigel ${ }^{1}$, M. Tegze ${ }^{1}$, M. Belakhovsky ${ }^{2}$ et O. Ulrich ${ }^{2}$ \\ Lawrence Berkeley National Laboratory, U.S.A. \\ ${ }_{1}^{1}$ Research Institute for Solid State Physics and Optics, Budapest, Hongrie \\ ${ }^{2}$ Service de Physique des Matériaux et Microstructures, CEA/Grenoble, France
}

\begin{abstract}
Résumé. De nouveaux progrès ont été obtenus dans la méthode d'holographie $\mathrm{X}$ par fluorescence (XFH), qui la font passer du stade de curiosité scientifique à celui d'un nouvel outil structural à l'échelle atomique. Grâce à la grande luminosité et stabilité de l'ESRF, ainsi qu'aux développements instrumentaux pour la détection ultrarapide, la XFH permet d'imager en une minute un groupe d'atomes autour de l'atome fluorescent. Quelques exemples de reconstruction d'images (atome léger, quasi-cristal, film mince) sont présentés.
\end{abstract}

\section{PROGRES EN HOLOGRAPHIE DE FLUORESCENCE X (XFH)}

A la suite des travaux pionniers [1-2] fondé sur le concept de source interne [3], nous illustrons ici les résultats de 3 années de développement de la méthode XFH sur l'European Synchrotron Radiation Facility (ESRF) à Grenoble. La mesure en XFH mode direct enregistre l'intensité de fluorescence tout autour de l'échantillon en fonction de sa direction d'émission, cf. Fig. 1a. L'hologramme est la très faible modulation d'amplitude (environ $10^{-3}$ ) de la fluorescence détectée sur une sphère de grand rayon centrée sur l'échantillon. Le processus de reconstruction permet de passer de cette mesure bidimensionnelle à l'arrangement spatial 3D des atomes voisins de l'atome fluorescent. Plus précisément, l'hologramme obtenu est moyenné sur tous les sites fluorescents d'un type atomique donné, comme pour la technique XAFS. Egalement comme en XAFS, la périodicité de translation n'est pas requise; c'est même une difficulté, car il en résulte des lignes de Kossel [4] qu'il faut ensuite éliminer. La méthode XFH inverse, par le théorème de réciprocité optique, l'atome émetteur devient un atome détecteur, et le taux de fluorescence reflète l'interférence entre l'onde plane incidente et ses diffusés par les atomes voisins, cf. Fig. $1 b$.

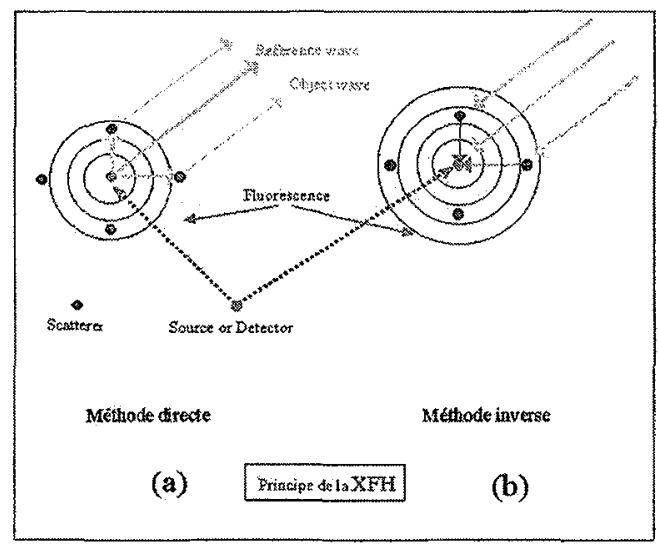

Fig. 1 : Principe de l'holographie de fluorescence $X$; méthodes directe et inverse 
Au plan expérimental, nous avons développé un montage permettant les 2 approches directe et inverse de la XFH [5]. La version actuelle comprend 3 rotations $\phi, \theta, 2 \theta$ permettant un balayage rapide et continu sur un angle solide approchant $2 \pi$. L'échantillon tourne sur lui-même à la fréquence azimutale $\phi$ de 10 $\mathrm{Hz}$; il est placé sur les cercles polaires concentriques $\theta, 2 \theta$, dont la vitesse de rotation est de 3 degrés par seconde, cf Fig. 2. Les balayages réalisés conduisent en fait à une contribution des 2 modes, directe et inverse, de la méthode XFH. La pratique a montré que les résultats sont meilleurs en raison d'une plus grande stabilité du montage, alors que l'élimination de la contribution de l'hologramme non désiré est aisée. A l'aide d'une électronique ad hoc et une mémoire tampon, chaque intervalle de mesure dure 200 $\mu$ seconde. Selon que l'on monochromatise le faisceau synchrotron ou que l'on utilise directement la source onduleur, le détecteur est respectivement une diode à avalanche (APD) ou une diode en mode photocourant, chacune avec son électronique associée. De plus, un analyseur d'énergie cylindrique de graphite a été conçu et placé devant le détecteur.

Le traitement des données est conduit à l'aide d'un logiciel que nous avons développé, détaillé dans [6]. Les différentes étapes [7] sont la soustraction du fond, la déconvolution de l'hologramme complémentaire, l'extension de l'hologramme à la sphère entière grâce aux symétries mesurées [4], le filtrage passe-bas et la reconstruction à partir de mesures à énergies multiples par une transformation de Helmholtz-Kirchhoff modifiée.

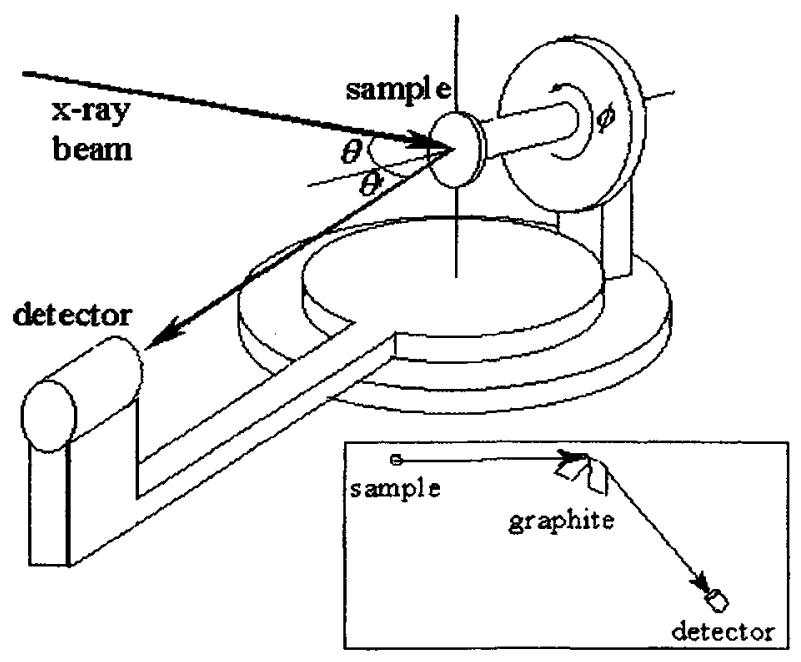

Fig. 2 : Schéma du montage expérimental

\section{CLUSTERS ATOMIQUES : CRISTAL MODELE ET PREMIERES APPLICATIONS}

Nous présentons dans cette section les résultats que nous avons obtenus, d'abord dans le cas de monocristaux parfaits, en vue de tester la méthode et ses performances en terme de résolution spatiale, de nombre de couches atomiques reconstruites, de taille d'atome etc. Puis nous aborderons les applications à des problèmes physiques pour lesquelles la XFH est susceptible d'apporter des informations nouvelles, tels que la structure locale des quasi-cristaux ou l'ordre directionnel, en relation avec les propriétés magnétiques, dans les couches minces épitaxiées.

\subsection{Monocristaux}

Nous avons étudié les monocristaux $\mathrm{CoO}$ et $\mathrm{NiO}$, d'orientation inhabituelle [111]. Dans le cas de $\mathrm{CoO}$, nous avions pu obtenir, pour la première fois, une résolution spatiale isotrope [8]. Récemment, nous avons également mesuré la fluorescence $\mathrm{K}$ du Ni d'une plaquette NiO[111] en XFH inverse [9], sur lignes 
onduleurs à l'ESRF. L'hologramme obtenu après traitement à $E=17.9 \mathrm{keV}$ est représenté sur la Fig.3a, en projetant l'hémisphère supérieur mesuré sur la surface plane de l'échantillon. Les hologrammes à 7 autres énergies sont similaires. Nous avons mesuré ces hologrammes avec une très bonne statistique. En effet, l'intensité des tâches atomiques est proportionnelle au carré de leur numéro atomique $Z$ et à l'inverse $\mathbf{r}^{-1}$ de leur distance à l'atome central. Nous avons calculé pour comparaison l'hologramme correspondant aux seuls atomes d'oxygène, Fig. $3 b$. On peut voir la différence d'échelle, qui permet de comprendre pourquoi jusqu'ici aucune image d'atome léger n'avait pu être obtenue. La Fig.4 montre à titre d'exemple la reconstruction selon les plans [100]; on notera que les mesures permettent de voir les atomes d'oxygène sur 2 couches de voisins, ainsi que des atomes de Ni très distants jusqu'à la $7^{\text {ème }}$ couche de coordination. Ce qui signifie que le volume imagé par holographie est d'environ 150 atomes. Les résultats obtenus sont doublement prometteurs : d'une part l'extension spatiale de l'image situe la méthode XFH entre la diffraction $\mathrm{X}$ classique, qui foumit l'ordre à longue distance et l'EXAFS qui donne l'ordre local; la XFH ayant une capacité complète d'imagerie $3 \mathrm{D}$; d'autre part, l'imagerie de l'oxygène ouvre la voie vers les applications biologiques.

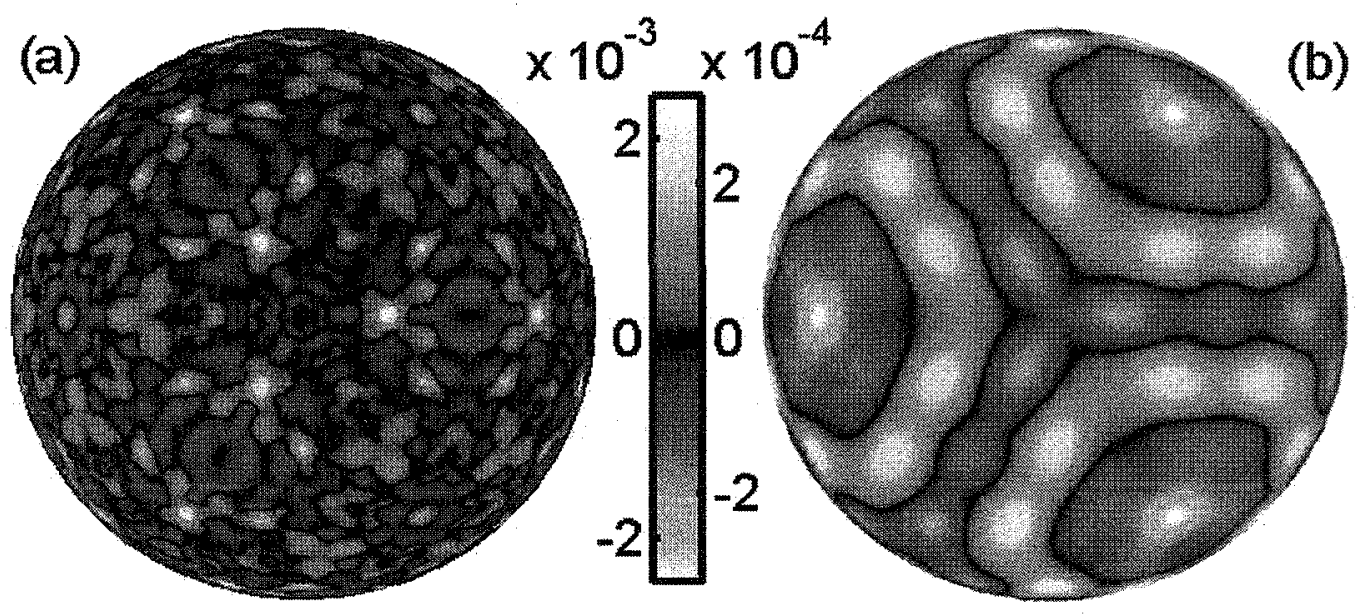

Fig. 3 : (a) Hologramme expérimental de NiO[111] ; (b) hologramme simulé des atomes d'oxygène

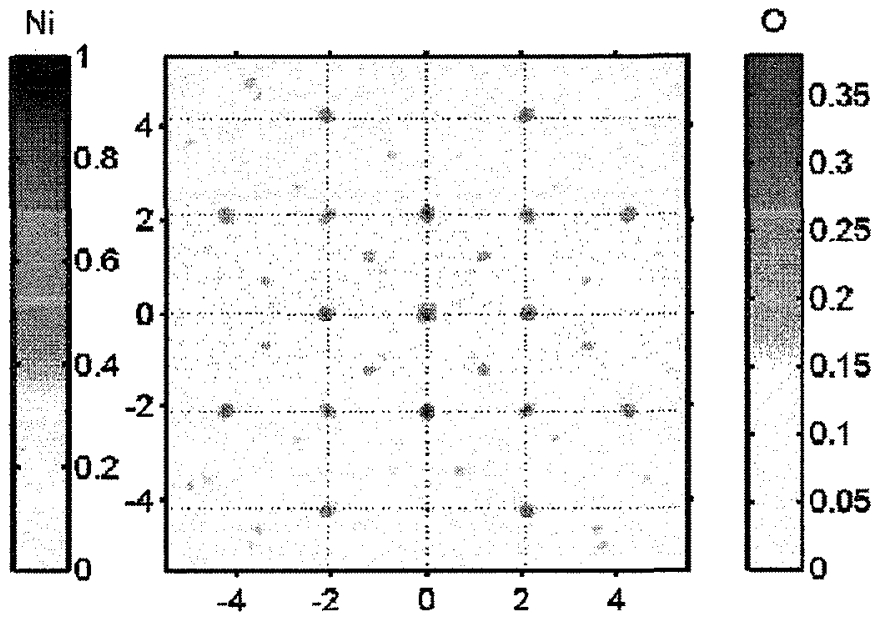

Fig. 4 : Reconstruction atomique dans le plan [100]; les atomes en bleu sont les $\mathrm{Ni}$, en jaune les $\mathrm{O}$ 


\subsection{Quasi-cristaux}

Les quasi-cristaux sont, à côté des solides cristallins et amorphes, un nouveau type de remplissage de l'espace, et présentent un ordre orientationel interdit dans les cristaux périodiques. La détermination de la décoration atomique des quasi-cristaux reste une question centrale. Les modèles classiques conduisent à l'existence d'un ordre local élevé, tant pour les distances que les orientations. Nous avons étudié le système de référence AIPdMn. L'application de la XFH à de telles structures; possible car elle ne requière pas la périodicité de translation, y présente un double intérêt : montrer l'efficacité de la méthode dans le cas d'environnements locaux différents d'une part, visualiser en 3D l'environnement d'un type d'atomes (ici $\mathrm{Al}, \mathrm{Pd}$ ou $\mathrm{Mn}$ ). Un disque de $20 \mathrm{~mm}$ et d'épaisseur $1 \mathrm{~mm}$ du quasi-cristal icosaédrique $\mathrm{Al}_{70,4} \mathrm{Pd}_{21} \mathrm{Mn}_{8,6}$ a été préparé par la méthode Czochralsky, avec l'axe d'ordre 5 normal à l'échantillon. L'expérience a été réalisée en XFH inverse en excitant par des photons de $16 \mathrm{keV}$ le seuil $\mathrm{K}$ du $\mathrm{Mn}(5,89 \mathrm{keV})$ sur ligne onduleur à l'ESRF. Après traitement du signal et reconstruction, on obtient l'hologramme de la Fig. 5 où la symétrie 5 est en évidence. La reconstruction présente 12 tâches de forte intensité situées aux sommets d'un icosaèdre. Cette expérience a pu être interprétée selon les modèles usuels. Ainsi, les tâches intenses proviennent d'une combinaison vers $4,56 \AA$ de la $5^{\text {ème }}$ couche de $\mathrm{Pd}$, de la $1^{\text {ère }}$ couche de Mn, et de la $3^{\text {ème }}$ de Al. Les couches plus proches, beaucoup moins occupées, ne sont pas imagées. Ces résultats [10] constituent à notre connaissance la première application de la XFH à un quasi-cristal.

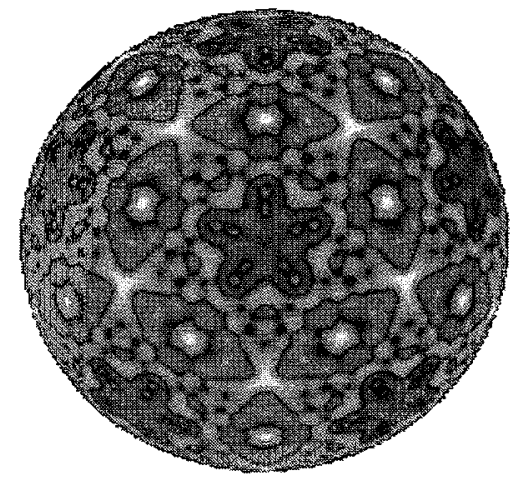

Fig. 5 : Hologramme de AlPdMn; la symétrie d'ordre 5 du quasi-crystal est apparente.

\subsection{Films épitaxiés}

Nous nous sommes aussi intéressés à l'étude des films minces épitaxiés, en étudiant les alliages magnétiques FePd et FePt. La motivation de l'étude est que ces matériaux présentent, selon les conditions de dépôt, et notamment de la température du substrat, un ordre chimique différent. Comme nous l'avons montré [11], l'anisotropie magnéto-cristalline et donc le magnétisme perpendiculaire dépend fortement de la nature de cet ordre chimique. Par conséquent la prédiction des propriétés magnétiques passe par la connaissance de l'arrangement atomique, la question posée étant celle du paramètre pertinent : ordre à longue distance (cf. diffraction classique), à très courte distance (XAFS) ou intermédiaire ? C'est pour étudier cette dernière hypothèse que nous avons appliqué la XFH au système FePt[100], sur lequel on peut mesurer soit l'environnement du Fe, soit celui du Pt. Une telle mesure présente un nouveau défi, car la quantité de matière est très faible dans les couches minces épitaxiées, d'où la nécessité d'un faisceau synchrotron intense. Nous présentons ici les résultats obtenus sur le seuil du Fe pour deux échantillons d'épaisseur $150 \mathrm{~nm}$ d'ordres chimiques différents : l'un proche de la phase $\mathrm{L} \mathrm{l}_{0}$, l'autre de la phase désordonnée $\gamma$. En combinant des mesures dans la gamme $15-19 \mathrm{keV}$, on obtient les hologrammes de la Fig. 6a,b respectivement dans le cas ordonné et désordonné. La simple observation montre qu'ils sont différents, et la symétrie d'ordre 4 est apparente. Nous avons de plus effectué la première mesure, à notre connaissance, en "faisceau rose", c'est-à-dire en illuminant l'échantillon sans monochromatisation, directement par un harmonique de la source onduleur. L'analyse de ces résultats est en cours. 


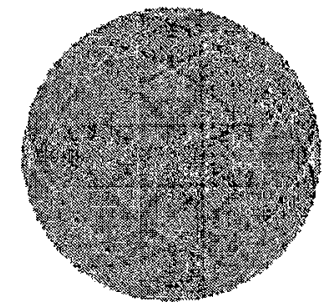

(a) ordonné

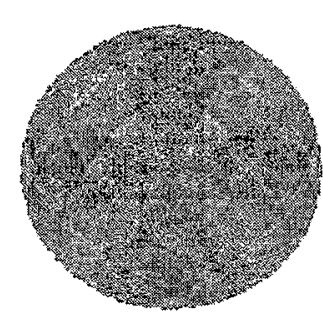

(b) désordonné

Fig. 6 : Hologrammes de couches minces de FePt : partiellement ordonné (a) et désordonné (b)

\section{Perspectives}

Les résultats présentés sont très prometteurs. Cependant, la XFH devra continuer d'être développée, tant du point de vue instrumental [7] que d'évaluation (obtention de la densité électronique). A terme, la XFH pourrait devenir une méthode structurale classique, s'intercalant pour le volume imagé entre l'EXAFS et la diffraction. Nous proposons en particulier qu'une station expérimentale dédiée soit prévue sur le synchrotron Soleil.

Les études futures pourraient concerner les couches minces en allant jusqu'aux couches interfaciales enterrées, les systèmes non périodiques (clusters orientés, quasi-cristaux), ainsi que les applications biologiques. La résolution temporelle et l'holographie $\gamma$ [12]. devraient aussi se développer.

\section{Remerciements}

Nous remercions A. Marty et B. Gilles pour les films épitaxiés, F. Schmithusen, J. Chevrier pour leur contribution à l'étude du quasi-crystal Nous remercions également l'ESRF pour son intérêt et son support constant, et tout particulièrement les équipes des lignes ID18, ID22 and ID32 pour leur aide au cours des expériences. Ce travail est soutenu par les programmes Balaton, OTKA, OMFB and EC-Biotechnology. L'un d'entre nous (S.M.) bénéficiait d'une bourse de l'U.E. n Erbflbict961366.

\section{Références}

1. M. Tegze \& G. Faigel, Europhys. Lett. 16, 41-46 (1991); M. Tegze \& G. Faigel, Nature 380, 49-51 (1996).

2. T. Gog et al. Phys. Rev. Lett. 76, 3132 (1996).

3. A. Szöke, A. in Short Wavelength Coherent Radiation: Generation and Applications (eds. Attwood, D.T. \& Boker, J.) 361-467 (AIP Conf. Proc. No. 147, American Institute of Physics, New York, 1986).

4. S. Marchesini, M. Belakhovsky, A.Q.R. Baron, G. Faigel, M. Tegze, Solid State Commun. 105 (1998) 685

5. S. Marchesini, O. Ulrich, G. Faigel, M. Tegze, M. Belakhovsky, A.S. Simionovici, Nuclear Instrument \& Methods (à paraître)

6. S. Marchesini, thèse, Universite Joseph Fourier, Grenoble, France, 2000 : adresse Web de la thèse : http://www-drfmc.ceng.cea.fr/Seminaires/index.htm

7. Faigel G. \& Tegze M. Rep. Prog. Phys. 62, 355-393 (1999)

8. M. Tegze, G. Faigel, S. Marchesini, M. Belakhovsky, A.I. Chumakov, Phys. Rev. Lett. 82, 4847-4850 (1991).

9. M. Tegze, G. Faigel, Stefano Marchesini, Michel Belakhovsky \& Olivier Ulrich, Nature (à paraître)

10. S. Marchesini, F. Schmithüsen, M. Tegze, G. Faigel, Y. Calvayrac, M. Belakhovsky, J. Chevrier, A.S. Simionovici (en préparation)

11. P. Kamp, A. Marty, B. Gilles, R. Hoffman, S. Marchesini, M. Belakhovsky, C. Boeglin, H.A. Dürr, G. van der Laan, S.S. Dhesi, A. Rogalev, Phys. Rev. B 50, 1105 (1999)

12. P. Korecki, J. Korecki, T. Slezak, Phys. Rev. Lett. 79, 3518 (1997) 